\title{
Attentional priorities and access to short-term memory: parietal interactions
}

Céline R. Gillebert ${ }^{a}$, Mads Dyrholm ${ }^{b}$, Signe Vangkilde ${ }^{b}$, Søren Kyllingsbæk ${ }^{b}$, Ronald Peeters ${ }^{c}$, Rik Vandenberghe ${ }^{a, d}$

${ }^{a}$ Laboratory for Cognitive Neurology, KU Leuven, Herestraat 49 - box 1022, 3000 Leuven, Belgium;

${ }^{b}$ Center for Visual Cognition, University of Copenhagen, Øster Farimagsgade 2A, 1353 Copenhagen K,

Denmark; ${ }^{c}$ Radiology Department, ${ }^{d}$ Neurology Department, University Hospitals Leuven, Herestraat 49

- box 7003, 3000 Leuven, Belgium

Correspondence to: Rik Vandenberghe. Neurology Department, University Hospitals Leuven, Herestraat 49 - box 7003, 3000 Leuven, Belgium. Phone: +3216344280, Fax: +3216344285. E-mail: rik.vandenberghe@uz.kuleuven. ac. be.

This is a pre-copy-editing, author-produced PDF of an article accepted for publication in NeuroImage following peer review. The definitive publisher-authenticated version (Gillebert, C.R., Dyrholm, M., Vangkilde, S., Kyllingsbæk, S., Peeters, R., and Vandenberghe, R. (2012). Attentional priorities and access to short-term memory: Parietal interactions. NeuroImage, 62:15511562) is available online at: http://dx.doi.org/10.1016/j.neuroimage.2012.05.038. 


\begin{abstract}
The intraparietal sulcus (IPS) has been implicated in selective attention as well as visual shortterm memory (VSTM). To contrast mechanisms of target selection, distracter filtering and access to VSTM, we combined behavioral testing, computational modeling and functional magnetic resonance imaging. Sixteen healthy subjects participated in a change detection task in which we manipulated both target and distracter set size. We directly compared the IPS response as a function of the number of targets and distracters in the display and in VSTM. When distracters were not present, the posterior and middle segments of IPS showed the predicted asymptotic activity increase with an increasing target set size. When distracters were added to a single target, activity also increased as predicted. However, the addition of distracters to multiple targets suppressed both middle and posterior IPS activity, thereby displaying a significant interaction between the two factors. The reverse interaction between target and distracter set size was significant in the right temporoparietal junction (TPJ), where activity was highest for a single target compared to any other condition. The interaction between target and distracter set size in IPS could not be accounted for by a simple explanation in terms of number of items accessing VSTM. Instead, it led us to a model where items accessing VSTM receive differential weights depending on their behavioral relevance, and secondly, a suppressive effect originates during the selection phase when multiple targets and multiple distracters are simultaneously present. This model reconciles the role of middle IPS in attentional selection and biased competition with its role in VSTM access.
\end{abstract}

\title{
Highlights
}

- IPS has been implicated both in attentional selection and visual short-term memory.

- We factorially varied target and distracter set size during change detection.

- A significant interaction occurred in middle IPS and right TPJ.

- Right TPJ was involved in spotting target singletons.

- The IPS profile reflected the combined effect of selection and VSTM access.

\section{Key words}

fMRI, visual attention, working memory, intraparietal sulcus, temporoparietal junction 


\begin{abstract}
Abbreviations
ANG, angular gyrus; DK rate, don't know rate; DMPFC, dorsomedial prefrontal cortex; EPI, echoplanar imaging; FEF, frontal eye fields; fMRI, functional magnetic resonance imaging; GLM, general linear model; IPS, intraparietal sulcus; OTS, occipitotemporal sulcus; RTs, reaction times; TPJ, temporoparietal junction; TVA, Theory of Visual Attention; VOI; volume-ofinterest; VSTM, visual short-term memory.
\end{abstract}

\title{
1 Introduction
}

Competition between targets and distracters for access to visual short-term memory (VSTM) is a central theme in the study of visual attention (Beck and Kastner, 2009; Bundesen, 1990; Bundesen et al., 2005; Desimone and Duncan, 1995; Duncan, 1996; Gazzaley, 2011; Huang and Pashler, 2007; Hung et al., 2005; Kastner and Ungerleider, 2001; Vandenberghe and Gillebert, 2009). Key phenomena underlying visual attention are the limited capacity available for processing information and the selectivity for goal-relevant information (Bundesen, 1990; Desimone and Duncan, 1995; Huang and Pashler, 2007). Studies which address limitations in VSTM capacity typically focus on the quantity of the information stored in VSTM, while keeping constant the separation of relevant from irrelevant information (Huang and Pashler, 2007). Activity in the intraparietal sulcus (IPS) correlates with the number of items in VSTM (Todd and Marois, 2004; Xu and Chun, 2006), and with interindividual differences in VSTM capacity $(K)$ (Todd and Marois, 2005). Conversely, studies which address the mechanisms of selection typically vary the quantity and the nature of distracters in the display, while keeping easy the access to the to-be-reported target (Huang and Pashler, 2007). When participants are asked to select a single target among competing distracters, activity in the middle IPS increases (Molenberghs et al., 2008; Vandenberghe et al., 2005), and relates in a linear mode to the number of items (target + distracters) in the display, up to a limit of four items (Mitchell and Cusack, 2008). The first objective of the current study was to evaluate the differential effect on IPS activity levels of increasing the number of targets versus distracters in the display.

Selection and access to VSTM can be modeled by the Theory of Visual Attention (TVA) (Bundesen, 1990; Bundesen et al., 2005; Bundesen and Habekost, 2008), a mathematical framework related to the biased competition account (Desimone and Duncan, 1995). TVA defines selection as a race between competing elements for access to VSTM, with the elements' processing speed being influenced by their attentional weight. According to TVA, the first $K$ elements 
that finish processing are stored in VSTM. TVA estimates individual attentional parameters, such as filtering efficiency and $K$, through the use of behavioral data (Habekost and Bundesen, 2003; Hung et al., 2005; Peers et al., 2005). These parameters can be applied to make single-trial predictions of the number of targets and distracters accessing VSTM (Dyrholm et al., 2011b). The second objective of this study was to compare the IPS response as a function of the number of targets and distracters accessing VSTM.

Within a TVA framework we have previously postulated that the middle segment of IPS is involved in attentional selection, i.e. the calibration of attentional weights (Molenberghs et al., 2008; Vandenberghe et al., 2005; Vandenberghe and Gillebert, 2009). This leads us to predict that the IPS activity would not only increase with increasing target set size (Todd and Marois, 2004), but also with increasing distracter set size. Such an activity increase in response to distracter set size (Mitchell and Cusack, 2008; Molenberghs et al., 2008; Vandenberghe et al., 2005 ) would result from an increased need for attentional selection. Alternatively, however, it could be explained within a 'VSTM storage' account: distracters may be stored in VSTM, even though subjects are instructed to ignore them (McNab and Klingberg, 2008; see also Lavie, 2005). TVA is particularly useful in this respect because it explicitly distinguishes between targets and distracters stored in VSTM.

Multiple studies have demonstrated that selective attention and VSTM interact at several stages during processing (Gazzaley, 2011; Lepsien et al., 2005; Lepsien and Nobre, 2007; Lepsien et al., 2011). Here we focused on interactions between attentional selection and access to VSTM. We used a change detection task (Cowan, 2001; Kyllingsbæk and Bundesen, 2009) and a fast event-related design with a very brief retention interval. We independently varied the difficulty of access to VSTM and that of selection through the number of simultaneously presented targets and distracters, respectively (Huang and Pashler, 2007). We directly compared the IPS response to an increasing target set size with its response to an increasing distracter set size. We predicted a main effect of both variables. Furthermore, to assess the differential effect of targets and distracters in VSTM, we modeled brain activity as a function of the number of targets or distracters accessing VSTM (latent variables predicted by TVA). By assessing IPS activity in response to target versus distracter set size in the display and in VSTM, we aimed to reconcile its proposed role in selection among competing stimuli (Molenberghs et al., 2007, 2008; Vandenberghe et al., 2005; Vandenberghe and Gillebert, 2009) with its role in access to VSTM (Todd and Marois, 2004; Xu and Chun, 2006). 


\section{Material and Methods}

\section{$2.1 \quad$ Subjects}

Sixteen healthy right-handed subjects (mean age 23.4 years, range 20-26 years, eight male and eight female) gave written informed consent in accordance with the Declaration of Helsinki. They had normal or corrected-to-normal vision, were free of psychotropic or vasoactive medication and had no neurologic or psychiatric history. Each of them performed the experimental paradigm in the MR scanner (event-related fMRI experiment) and in a psychophysical testing room (behavioral experiment) on two different days within three weeks. The behavioral experiment always followed the fMRI experiment. The study was approved by the Ethics Committee, University Hospitals Leuven.

\subsection{Stimuli and experimental paradigm}

Stimulus presentation and response registration were controlled by a PC running Presentation 11.3 (Neurobehavioral systems, Albany, CA).

\subsubsection{Event-related fMRI experiment}

Stimuli were projected onto a translucent screen in front of the subject during scanning by means of a liquid crystal display projector (1024×768 pixels; Barco 6400i; Barco, Kortrijk, Belgium). Eye movements were registered using an Applied Science Laboratory infrared system (ASL 5000/LRO system; Waltham, MA) and stored for subsequent analysis.

Subjects performed a single-probe change detection task under covert attention conditions (Cowan, 2001). A trial started with a temporal warning cue (duration $200 \mathrm{~ms}$ ) (Fig. 1A). After a short delay (300 ms), a display was presented, showing 1 to 12 white characters (digits and capital letters, size $0.75^{\circ}$ ) against a black background. The 12 possible stimulus locations were equally spaced on an imaginary circle centered on a fixation point at an eccentricity of $4.45^{\circ}$. None of the 12 equidistant locations were occupied by more than one character. The stimulus display was terminated after $200 \mathrm{~ms}$ by an effective mask display presented for $300 \mathrm{~ms}$. The mask display showed 12 patterned masks, one in each of the 12 locations. Each pattern was equal in size to a stimulus character and consisted of jumbled letter features. We independently manipulated the number of targets (target set size: 1T, 2T, $4 \mathrm{~T}$ or $6 \mathrm{~T}$ ) and the number of distracters (distracter set size: 0D, 2D, 4D or 6D) in the display. The selection criterion was based on alphanumerical class (Shibuya and Bundesen, 1988): letters were to be remembered 
and digits to be ignored. The identity of the targets and distracters was determined by drawing at random from a set of consonants (BCDFGHJKLMPQRSTVXZ) and digits (excluding 0), respectively. The spatial distribution of targets and distracters over the 12 locations was random with the constraint that the number of targets in the left hemifield was identical to the number of targets in the right hemifield. The same constraint was applied for the distracters. In trials with only one target, the target was presented equally often in the left and the right visual field. The mask was followed by a brief delay $(200 \mathrm{~ms})$ during which only the fixation point was shown. Next, a single probe letter was shown at one of the locations previously occupied by a letter $(200 \mathrm{~ms})$. In half of the trials, the letter probe was identical to the corresponding letter in the preceding display and in half it was different. Participants were instructed to determine whether the probe letter was identical to the letter at the same location in the stimulus display. Participants held a response box with multiple response buttons in their right hand. Three different responses were allowed: 'change', 'no change' and 'don't know' (unforced choice procedure, Kyllingsbæk and Bundesen, 2009). The intertrial interval was fixed to 3000 ms. Each subject performed 768 trials in total, 48 trials per condition, divided into 6 runs of 128 trials containing 8 trials per condition. In each run, the order of the 16 conditions was pseudo-randomized (Wager and Nichols, 2003) and task trials were intermixed with 22 null events.

\subsubsection{Behavioral experiment}

Participants were seated at $50 \mathrm{~cm}$ from a 19-inch cathode ray tube monitor (resolution $1024 \times 768$ pixels, refresh rate $75 \mathrm{~Hz}$ ) in a semidarkened room. Gaze fixation was monitored on-line by means of infrared eye monitoring (ViewPoint Eye Tracker; Arrington Research; Scottsdale, AZ).

The stimuli and task were identical to those used in the fMRI experiment, with the exception of exposure duration $(13,40,80,120,160$ or $200 \mathrm{~ms})$ in the conditions without distracters. This additional manipulation enabled us to estimate individual attentional parameters related to processing speed and increased the accuracy of the estimates of other attentional parameters (Shibuya and Bundesen, 1988). The manipulation of target set size, distracter set size and exposure duration in conditions without distracters resulted in 36 unique conditions. Each subject performed 1008 trials in total, divided into 7 blocks of 144 trials containing 4 trials per condition. Within each run, the order of the 36 conditions was randomized. 

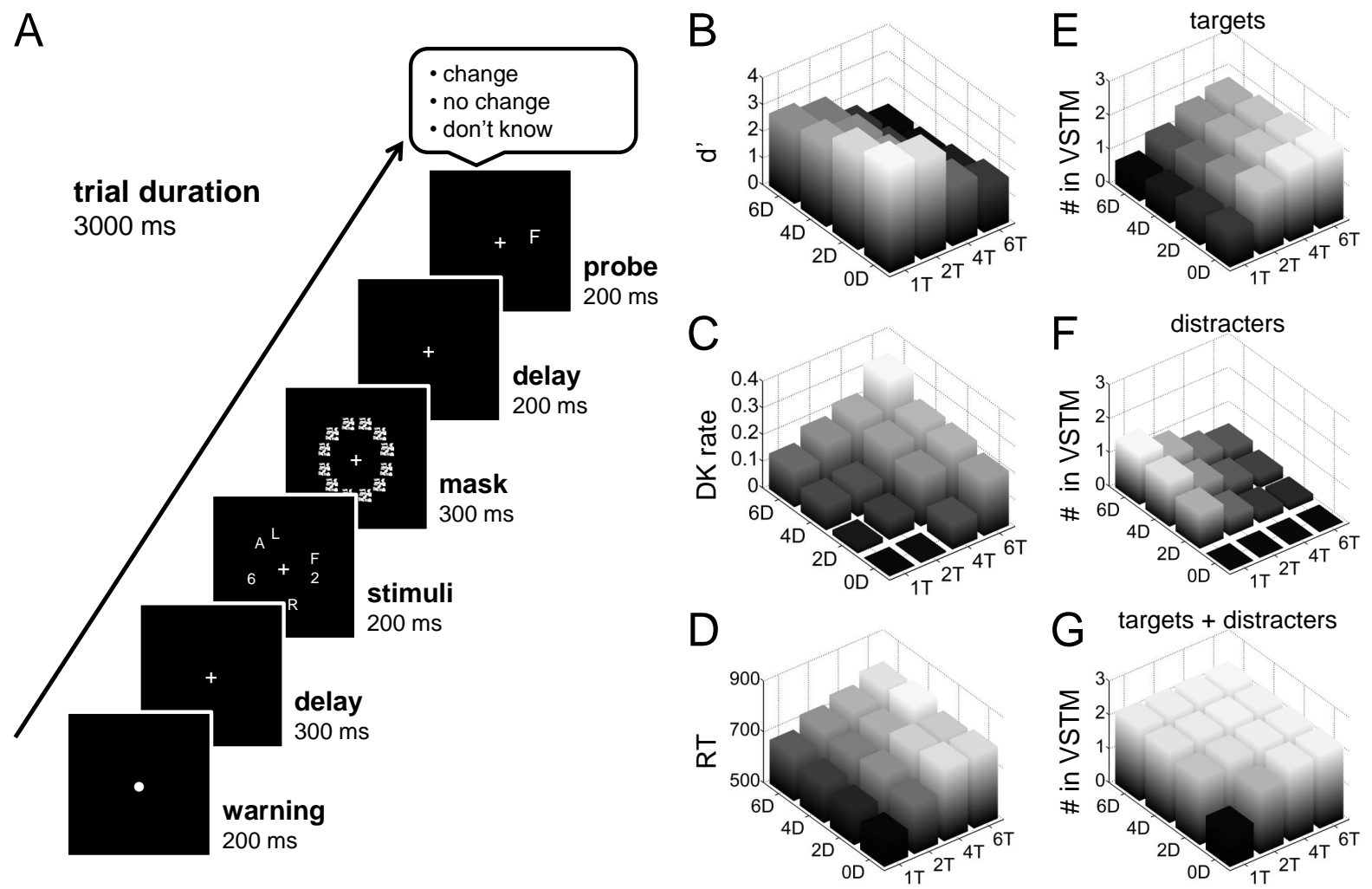

Figure 1 - Stimuli, task and behavioral performance (A) Each trial began with a warning cue. Next, subjects viewed a masked stimulus display containing 1 to 6 targets (letters) to be stored in VSTM and 0 to 6 distracters (digits) to be filtered out. Following a brief delay, subjects indicated by a key press whether a probe letter was identical to the target letter presented at the same location in the stimulus display. (B-D) Average d' score (B), don't know (DK) rate $(\mathbf{C})$ and RT $(\mathbf{D})$ in each cell of the $4 \times 4$ factorial design. The bars are grey-scaled according to the performance. (E-G) The average number of targets, distracters and items (targets + distracters) in VSTM, for each condition in the $4 \times 4$ factorial design. The bars are grey-scaled according to the number of items. Abbreviations: $\mathrm{T}=$ targets; $\mathrm{D}=$ distracters 


\section{$2.3 \quad$ Image acquisition}

The fMRI experiment was run in a 3T Philips Intera magnet with an eight-channel sensitivity encoding (SENSE) head coil. The whole-brain functional scans consisted of $\mathrm{T}^{*}$ gradient-echo echoplanar images (EPI) acquired continuously in an ascending order (6 time series, 225 scans per time series, $2000 \mathrm{~ms}$ repetition time (TR), $30 \mathrm{~ms}$ echo time (TE), $80 \times 80$ acquisition matrix, $2.75 \times 2.75 \mathrm{~mm}^{2}$ in-plane resolution, $363.75 \mathrm{~mm}$ thick axial slices without gap). We also acquired a T1-weighted anatomical image (9.6 ms TR, $4.6 \mathrm{~ms}$ TE, $256 \times 256$ acquisition matrix, $1 \times 1 \mathrm{~mm}^{2}$ in-plane resolution, $1821.2 \mathrm{~mm}$ thick coronal slices).

\subsection{Data analysis}

\subsubsection{Analysis of behavioral data in the fMRI and behavioral experiments}

Analysis of performance across conditions We determined the proportion of 'don't know' trials in each condition. Using the remaining trials, we calculated d', a bias-independent measure of performance (Macmillan and Creelman, 1991). Trials with reaction times (RTs) more than 3 standard deviations apart from the mean RT of a subject were excluded from the analysis. Don't know rates (DK rates), d' scores and RTs on correct trials were submitted to a $4 \times 4$ analysis of variance (ANOVA) with target set size (1T, 2T, 4T, 6T) and distracter set size $(0 \mathrm{D}, 2 \mathrm{D}, 4 \mathrm{D}, 6 \mathrm{D})$ as within-subject factors. When sphericity could not be assumed (Mauchly's sphericity test: $P<0.05), P$-values were adjusted using the Greenhouse-Geisser correction $(\mathrm{G}-\mathrm{G}$ adj.).

Analysis of eye movements We defined a region on the display covering the stimulus locations from $3^{\circ}$ to $7^{\circ}$ eccentricity. Deviations of eye movements into that region were detected automatically (Molenberghs et al., 2008) and submitted to a $4 \times 4$ ANOVA with target set size and distracter set size as within-subject factors.

Estimation of individual attentional parameters Five attentional parameters were estimated using TVA-based modeling (Kyllingsbæk, 2006; Dyrholm et al., 2011a) (LIBTVA software, http://zappa.psy.ku.dk/libtva): (1) $t_{0}$, the threshold for conscious perception measured in milliseconds, (2) $C$, the speed of visual processing measured in number of elements processed per second, (3) $K$, the capacity of VSTM measured in number of elements, (4) $\alpha$, the filtering efficiency defined as the attentional weight of a distracter divided by the attentional weight of a target, where $\alpha$ values close to 0 indicate efficient selection of targets and values 
close to 1 indicate unselective processing, and (5) $\omega$, one attentional weight per quadrant of the visual field, each representing the weight of a target shown in the respective quadrant $(\alpha \omega=$ the attentional weight of a distracter). The attentional parameters were estimated separately for the behavioral and fMRI experiment using Maximum Likelihood estimation. Since exposure duration was only varied in the behavioral experiment, estimation of $K, \alpha$ and $\omega$ in the fMRI experiment was constrained by the $t_{0}$ and $C$ estimates from the behavioral experiment. A detailed description of the estimation procedure is provided in the Supplementary materials.

TVA-based single-trial predictions We estimated two latent variables related to the attentional state of each subject on each trial, using his or her attentional parameters derived from the fMRI study (Dyrholm et al., 2011b): (1) the number of targets selected and stored in VSTM and (2) the number of distracters selected and stored in VSTM. The procedure for deriving these latent variables is described in the Supplementary materials.

\subsubsection{Analysis of task-related fMRI data}

Preprocessing Preprocessing and statistical analysis were carried out with Statistical Parametric Mapping 5 (Welcome Trust Centre for Neuroimaging, London, UK, http://www.fil. ion.ucl.ac.uk/spm). The EPI images were corrected for differences in acquisition time, realigned to correct for head movements and co-registered to the T1-weighted image. The T1weighted image was warped into the Montreal Neurological Institute (MNI) space (via the segmentation option in SPM) and the resulting transformation was used to spatially normalize the functional images. The voxel size of the images in MNI space was $3 \times 3 \times 3 \mathrm{~mm}^{3}$. The images were spatially smoothed with a $5 \times 5 \times 7 \mathrm{~mm}^{3}$ full-width half-maximum kernel. For each of the analyses, we included the 6 motion regressors and a dummy regressor per run. 'Don't know' trials were modeled as separate regressor in each analysis.

Targets and distracters in the stimulus display To evaluate the IPS response as a function of the number of targets and distracters in the display, we modeled the fMRI data using a general linear model (GLM) with 16 main regressors, coding for each combination of target $(1 \mathrm{~T}, 2 \mathrm{~T}, 4 \mathrm{~T}, 6 \mathrm{~T})$ and distracter $(0 \mathrm{D}, 2 \mathrm{D}, 4 \mathrm{D}, 6 \mathrm{D})$ set size. The main effects and the interaction effect were assessed at the second-level of analysis with $F$ contrasts and a twoway within-subjects ANOVA implemented in SPM. Main results are reported at $P<0.05$ with family-wise error (FWE) correction for multiple comparisons at the voxel-level. For clusters 
of activations in a priori regions of interests, i.e. the middle and posterior segments of IPS (Molenberghs et al., 2008; Todd and Marois, 2004; Vandenberghe et al., 2005; Xu and Chun, 2006), the threshold was lowered to $P=0.001$ (uncorrected) and a cluster extent of 5 voxels.

Given the complexity of a $4 \times 4$ factorial design and the need to evaluate the origin of any interaction effects in a structured manner, we reduced it to a $2 \times 2$ design. We modeled the fMRI data using a GLM with 4 main regressors, coding for each combination of the difficulty of access to VSTM (difficult access versus easy access: $>1 \mathrm{~T}$ versus $1 \mathrm{~T}$ ) and the difficulty of selection (difficult selection versus easy selection: $\geq 1 \mathrm{D}$ versus $0 \mathrm{D}$ ). We calculated the following contrasts:

- main effect of difficulty of access to VSTM: >1T minus $1 \mathrm{~T}$

- main effect of difficulty of selection: $\geq 1 \mathrm{D}$ minus $0 \mathrm{D}$

- interaction between difficulty of selection and difficulty of access to VSTM, and the reverse: $(>1 \mathrm{~T}, 0 \mathrm{D}$ minus $1 \mathrm{~T}, 0 \mathrm{D})$ minus $(>1 \mathrm{~T}, \geq 1 \mathrm{D}$ minus $1 \mathrm{~T}, \geq 1 \mathrm{D})$

Contrast images were calculated separately for each subject and evaluated at the second-level using one-sample $t$ tests (random effects analysis, threshold: voxel-level uncorrected $P<0.001$, cluster-level FWE-corrected $P<0.05$ (Poline et al., 1997)). The response profile of clusters showing a significant interaction effect was characterized by means of 4 post-hoc contrasts on the peak \% signal change, averaged across all voxels in the cluster (one-tailed paired-sample $t$ tests): (1) the simple effect of adding targets in trials without distracters ( $>1 \mathrm{~T}, 0 \mathrm{D}$ minus 1T,0D); (2) the simple effect of adding distracters in trials with one target (1T, $\geq 1 \mathrm{D}$ minus $1 \mathrm{~T}, 0 \mathrm{D})$; (3) the simple effect of adding targets in trials with distracters $(>1 \mathrm{~T}, \geq 1 \mathrm{D}$ minus $1 \mathrm{~T}, \geq 1 \mathrm{D})$; (4) the simple effect of adding distracters in trials with more than one target $(>1 \mathrm{~T}, \geq 1 \mathrm{D}$ minus $>1 \mathrm{~T}, 0 \mathrm{D})$. Statistical significance was Bonferroni-corrected for multiple comparisons (corrected $P<0.05)$.

TVA-based estimates of targets and distracters accessing in VSTM We determined the relationship between brain activity and targets and distracters accessing VSTM in two ways. First, to assess the IPS response as a function of the number of items in VSTM, the fMRI data were modeled using the TVA-based single-trial predictions (latent variables) of the number of targets and distracters accessing VSTM as parametric regressors (Büchel et al., 1998). For each subject, we created whole-brain contrast images testing for the intra-individual linear relationship between the fMRI activity and the two parametric modulators. As our first regressor, we entered the number of targets accessing VSTM and as our second regressor, the 
number of distracters accessing VSTM. Note that these two variables are highly correlated and that SPM will estimate which portion of the experimental variance can be attributed to the first regressor and which portion of the remaining variance to the second. These contrast images were evaluated at the second-level using one-sample $t$ tests (random effects analysis, threshold: voxel-level uncorrected $P<0.001$, cluster-level FWE-corrected $P<0.05$ (Poline et al., 1997)). The effect size of the parametric modulators were plotted by means of rfxplot (Gläscher, 2009) (http://rfxplot.sourceforge.net), which is capable of dividing a parametric modulator into different bins containing an equal number of trials, and estimating the average $\%$ signal change for each bin.

As our second approach, we constructed a general linear model with as independent variables the TVA-based predictions of targets and distracters accessing VSTM for each subject and for each of the 16 conditions in the $4 \times 4$ factorial design, averaged over trials (Fig. 1E-F). The dependent variables were the right and left middle IPS peak \% signal change for each of the 16 conditions (Fig. 2D-E). Left and right middle IPS were defined based on the interaction between the number of targets and the number of distracters in the display (Fig. 2C, voxel-level uncorrected $P<0.001$, cluster extent 5). Our generalized linear model consisted of the linear combination $b_{0}+b_{1} \mathrm{E}[$ Number of targets in VSTM $]+b_{2} \mathrm{E}[$ Number of distracters in VSTM] with a logarithmic link function and Normal noise distribution. The coefficients $b_{0}, b_{1}$, and $b_{2}$ then represented activity response per baseline, target, and distracter, respectively. For each subject, we estimated the coefficients $b_{0}, b_{1}$, and $b_{2}$. We also computed the residual per condition.

Analysis of interindividual differences in attentional parameters We investigated whether interindividual differences in VSTM capacity (Todd and Marois, 2005) and filtering efficiency correlated with interindividual differences in brain activity. We calculated the Pearson's correlation between $K$ (VSTM capacity) and the peak \% signal change in the condition containing a maximum proportion of targets (6T0D), and between $\alpha$ (filtering efficiency) and the peak \% signal change in the condition containing a maximum proportion of distracters (1T6D). The analysis was restricted to brain areas showing a positive correlation with the number of targets or distracters accessing VSTM, respectively. The statistical threshold was set at $P<0.05$, Bonferroni-corrected for the number of brain areas.

Control analyses In a first control analysis, we evaluated the consistency of our results with neuroimaging studies reported in the literature, which manipulated either the difficulty of access 
A
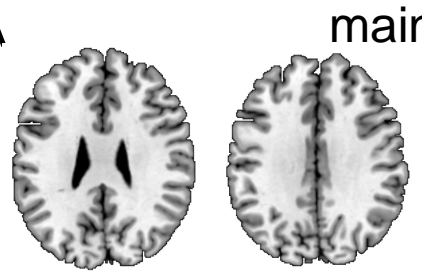

main effect of target set size

B
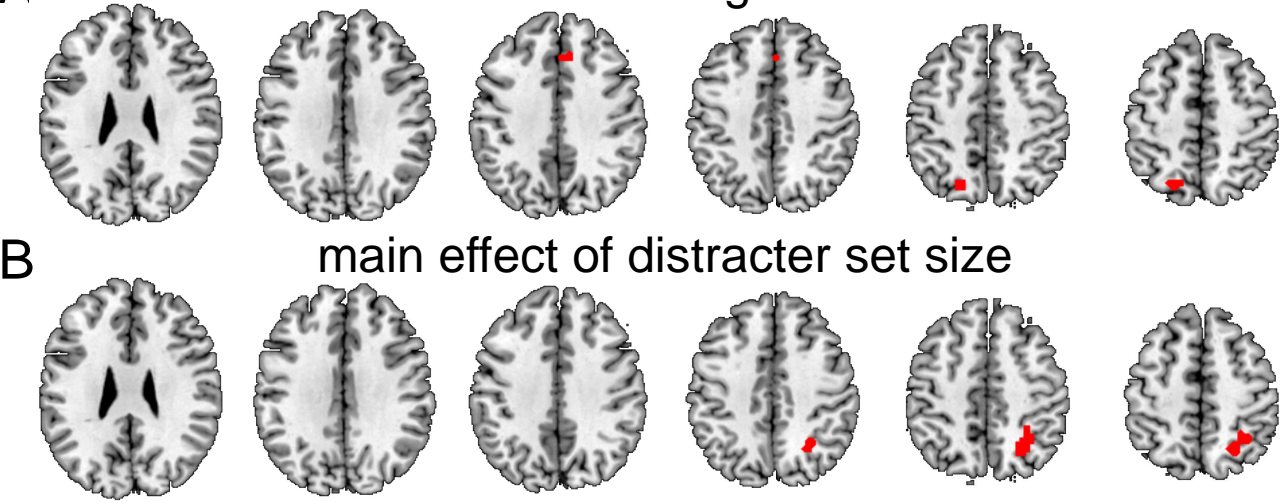

main effect of distracter set size

C
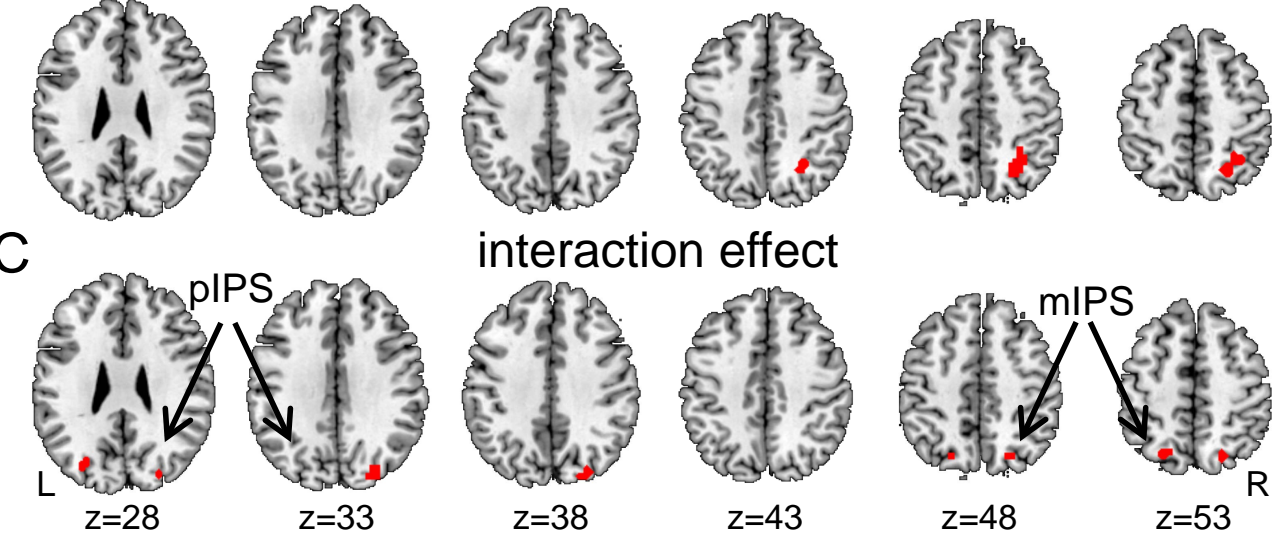

interaction effect
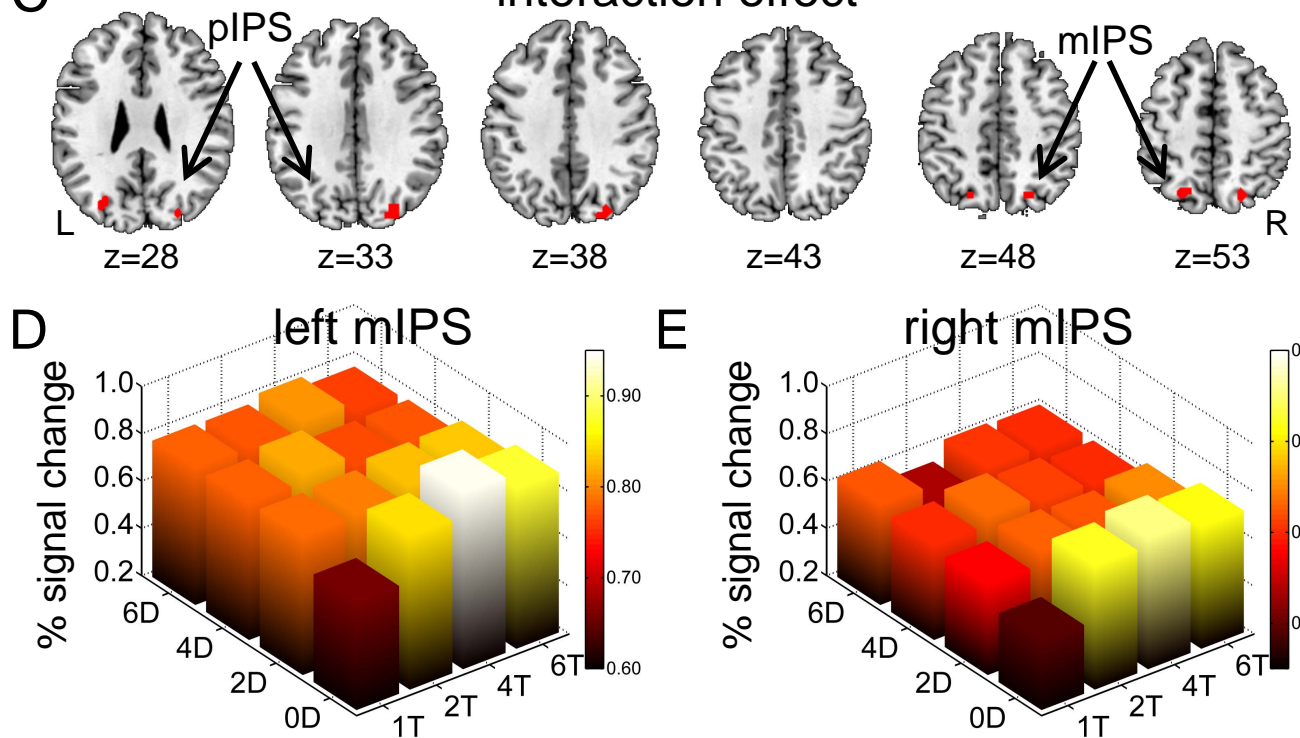

$\mathrm{E}$
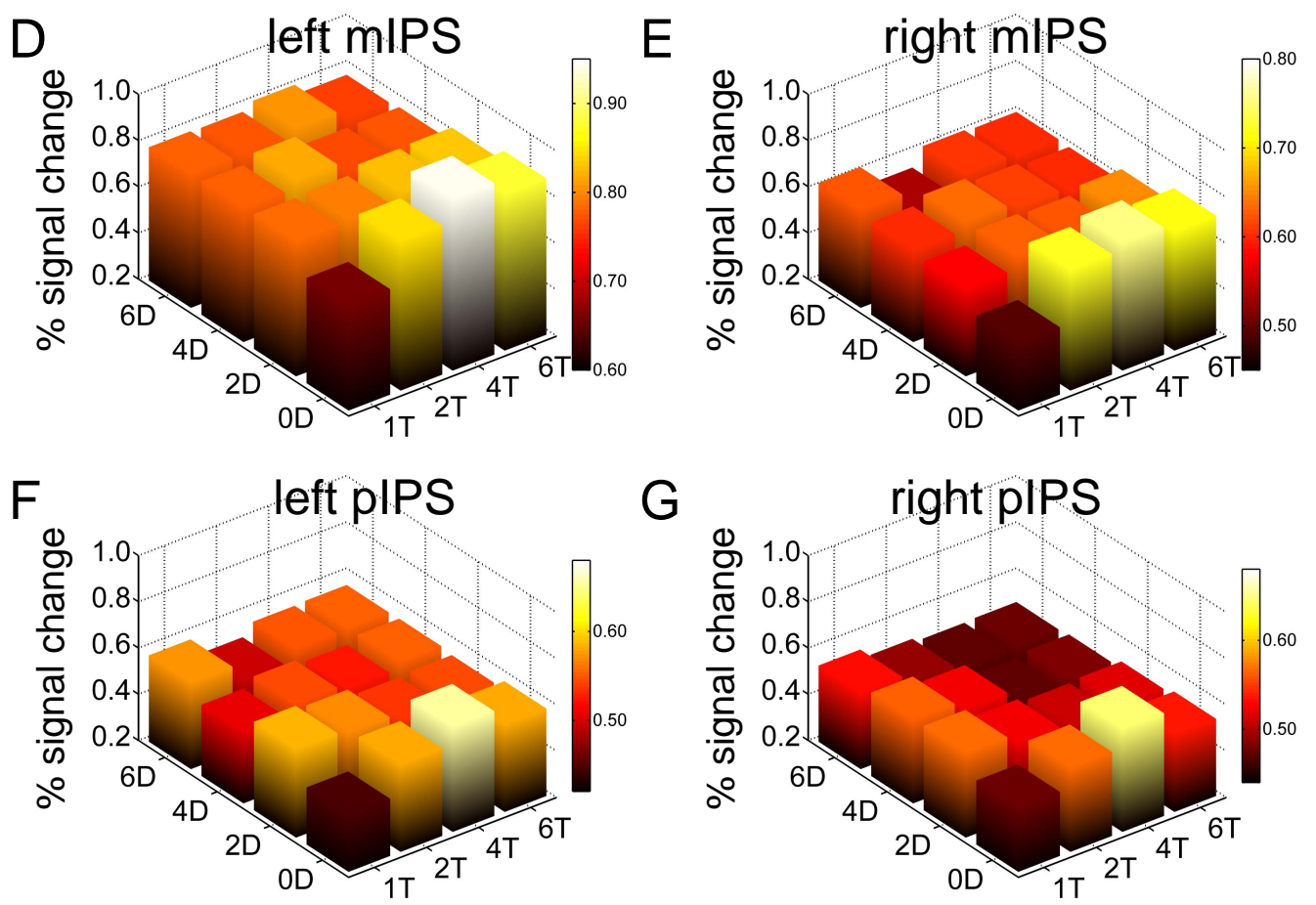

Figure 2 - Activity modulation by target and distracter set size (A-C) F-maps for the main effect of target set size (A), the main effect of distracter set size (B) and the interaction between target set size and distracter set size (C), projected onto axial slices of the brain. (D-G) Peak \% signal change for each condition in the $4 \times 4$ factorial design, averaged over all voxels belonging to the middle segment of left (D) and right (E) IPS, and the posterior segment of left $(\mathbf{F})$ and right (G) IPS. The bars are color-scaled according to the response amplitude. Abbreviations: IPS, intraparietal sulcus. 
to VSTM (Todd and Marois, 2004; Xu and Chun, 2006) or the difficulty of selection (Molenberghs et al., 2008; Vandenberghe et al., 2005). Using a random effects analysis (threshold: voxel-level uncorrected $P<0.001$, cluster-level FWE-corrected $P<0.05$ (Poline et al., 1997)), we identified the effect of an increasing target set size in conditions without distracters $(>1 \mathrm{~T}, 0 \mathrm{D}$ minus 1T,0D) and also determined which brain areas were sensitive to the presence of competing distracters when only one target was present in the display (1T, $\geq 1 \mathrm{D}$ minus $1 \mathrm{~T}, 0 \mathrm{D})$.

In a second control analysis, we compared the response to correct "change" trials versus correct "no change trials" (random effects analysis, threshold: voxel-level uncorrected $P<0.001$, cluster-level FWE-corrected $P<0.05$ (Poline et al., 1997)).

In a third control analysis, we evaluated in a whole-brain analysis the effect of increasing number of stimuli on display (random effects analysis, parametric modulation, threshold: voxellevel uncorrected $P<0.001$, cluster-level FWE-corrected $P<0.05$ (Poline et al., 1997)).

\section{Results}

\subsection{Behavioral results}

\subsubsection{Performance across conditions}

We examined the behavioral performance during the fMRI experiment by computing a repeated measures ANOVA with 4 levels of target set size and 4 levels of distracter set size (Fig. 1B-D). We observed a significant main effect of target set size (d': $F_{3,45}=163.01, P<0.0001$; don't know (DK) rate: $F_{1.1,17.2}=20.91$, G-G adj. $P=0.0002$; RTs: $F_{1.4,20.6}=56.58, \mathrm{G}-\mathrm{G}$ adj. $P<0.0001$ ), a significant main effect of distracter set size (d': $F_{3,45}=43.06, P<0.0001$; DK rate: $F_{1.4,21.7}=20.02, \mathrm{G}-\mathrm{G}$ adj. $P<0.0001$; RTs: $\left.F_{3,45}=2.93, P=0.04\right)$ and a significant interaction between target set size and distracter set size (d': $F_{9,135}=2.56, P=0.01$; DK rate: $F_{3.7,55.5}=3.08$, G-G adj. $P=0.03$; RTs: $F_{3.1,46.2}=4.95, P=0.004$ ) (Fig. 1B-D).

The average number of eye movements towards the peripheral stimuli (mean \pm standard error of the mean: $0.32 \pm 0.08)$ did not depend on target set size, distracter set size or on the interaction $(P>0.18)$.

The behavioral experiment (Supplementary Table 1) confirmed a significant main effect of target set size (d': $F_{3,42}=126.07, P<0.0001$; DK rate: $F_{1.2,18.4}=29.42, \mathrm{G}-\mathrm{G}$ adj. $P<0.0001$; RTs: $F_{1.6,24.5}=47.59, \mathrm{G}-\mathrm{G}$ adj. $P<0.0001$ ), a significant main effect of distracter set size (d': $F_{3,42}=26.66, P<0.0001 ;$ DK rate: $F_{1.5,22.1}=23.78$, G-G adj. $P<0.0001$; RTs: $F_{3,45}=5.77$, 
$P=0.002)$ and a significant interaction between target set size and distracter set size (d': $F_{9,126}=4.80, P<0.0001 ;$ DK rate: $F_{4.8,71.6}=2.40, \mathrm{G}-\mathrm{G}$ adj. $P<0.05$; RTs: $F_{3.9,58.8}=2.22, \mathrm{G}-\mathrm{G}$ adj. $P=0.08)$.

\subsubsection{Individual attentional parameters}

We estimated several attentional parameters for each subject using TVA. The estimated capacity of $\operatorname{VSTM}(K)$ was $2.62 \pm 0.18$ in the behavioral experiment and $2.23 \pm 0.08$ in the fMRI experiment. On average, subjects assigned a lower attentional weight to distracters than to targets ( $\alpha$ towards zero), both in the behavioral $(\alpha: 0.28 \pm 0.04)$ and in the fMRI experiment ( $\alpha: 0.24 \pm 0.03$ ). The correlations of the individual parameters between the behavioral and the fMRI session were significant for $K(r=0.55, P=0.03)$ and for $\alpha(r=0.52, P=0.04)$. We used the parameters derived from the fMRI study to compute for each subject the predicted number of targets (Fig. 2E) and distracters (Fig. 2F) accessing VSTM in each trial (see Supplementary Fig. 1A-B for an example).

\subsection{Task-related functional MRI}

\subsubsection{Targets and distracters on display}

The main effect of target set size was significant in the middle segment of left IPS ( $\mathrm{x}, \mathrm{y}, \mathrm{z}=-21$, $-69,51, Z=4.33$, extent 14 voxels at $3 \times 3 \times 3 \mathrm{~mm}^{3}$; uncorrected $\left.P<0.001\right)$ and the dorsomedial prefrontal cortex (DMPFC) $(9,27,36 ; Z=5.16$; extent 8; whole-brain FWE-corrected $P=0.005)$ (Fig. 2A). The main effect of distracter set size was significant in the middle segment of right IPS (27, $-54,48, Z=4.27$, extent 39; uncorrected $P<0.001)$ (Fig. 2B). The interaction between target set size and distracter set size was significant in the middle and posterior segment of left and right IPS (Fig. 2C-D; -24, -66, 51; $Z=5.32$; extent 8; whole-brain FWE-corrected $P=0.002$ )(Fig. 2C,E; 24, -69, 51; $\mathrm{Z}=3.35$; extent 6 ; uncorrected $P<0.001$ )(Fig. 2C,F-G; -30, -78, 27; $Z=3.70$; extent 11 ; uncorrected $P<0.001 ; 27,-84,36 ; Z=3.94$; extent 15 ; uncorrected $P<0.001)$.

When the data were analyzed according to a $2 \times 2$ design, a similar pattern was observed: increasing difficulty of access to VSTM ( $>1 \mathrm{~T}$ versus $1 \mathrm{~T})$ increased activity in left middle IPS (-21, -69, 51; $Z=4.09$; extent 40; cluster-level FWE-corrected $P=0.001)$, right frontal eye fields (FEF) $(24,-3,51 ; Z=4.59$; extent 48; $P<0.001)$ (Fig. 3A, black outline) and DMPFC (9, $27,33 ; Z=4.84 ;$ extent $129 ; P<0.001)$. Increasing difficulty of visual selection $(\geq 1 \mathrm{D}$ versus 
$0)$ increased activity in $\operatorname{DMPFC}(-3,33,39 ; Z=4.18$; extent $30 ; P=0.001)$. The interaction between difficulty of selection and difficulty of access to VSTM was significant in left and right middle and posterior IPS (Fig. 3A; Table 1A): Activity in middle (Fig. 3B-C) and posterior (Fig. 3D-E) segments of left and right IPS increased as a function of target set size when no distracters were presented $(>1 \mathrm{~T}, 0 \mathrm{D}$ in red versus $1 \mathrm{~T}, 0 \mathrm{D}$ in blue). However, this effect disappeared in the presence of multiple distracters $(>1 \mathrm{~T}, \geq 1 \mathrm{D}$ in magenta versus $1 \mathrm{~T}, \geq 1 \mathrm{D}$ in cyan). Likewise, increasing distracter set size increased activity in middle and posterior IPS bilaterally in the case of a single target $(1 \mathrm{~T}, \geq 1 \mathrm{D}$ in cyan versus $1 \mathrm{~T}, 0 \mathrm{D}$ in blue), but this effect disappeared when multiple targets were present $(>1 \mathrm{~T}, \geq 1 \mathrm{D}$ in magenta versus $>1 \mathrm{~T}, 0 \mathrm{D}$ in red) (Fig. 3B-E; Table 1A). The same effects were observed in the left occipitotemporal sulcus (OTS) (Fig. 3F-G).

The inverse interaction was significant in the right temporoparietal junction (TPJ) (Fig. 3A): activity was higher for a single target without distracters than for multiple targets and/or distracters (Fig. 3H-I; Table 1B).

To evaluate any differences between left and right middle and posterior IPS (Fig. 3B-C; Fig. 3D-E), we defined volumes-of-interest (VOIs) based on significant clusters of activity in the interaction contrast (Table 1A). We analyzed the peak \% signal change in these VOIs using an ANOVA with hemisphere (left versus right), VOI (posterior IPS versus middle IPS), difficulty of selection $(\geq 1 \mathrm{D}$ versus $0 \mathrm{D})$ and difficulty of access to VSTM $(>1 \mathrm{~T}$ versus $1 \mathrm{~T})$ as within-subject factors. We observed a significant three-way interaction between VOI, difficulty of selection and difficulty of access to $\operatorname{VSTM}\left(F_{1,15}=10.8, P=0.005\right)$, which did not depend on hemisphere $(P=0.93)$. In the absence of distracters, increasing target set size yielded a stronger activity increase in middle compared to posterior IPS $(P=0.001)$, but had a similar effect on middle and posterior IPS activity when distracters were present $(P=0.15)$ (Fig. 3B-E).

To evaluate the absence of an effect of early visual cortex activity, we lowered the significance threshold to an uncorrected $P<0.01$. None of the contrasts reached significance in early visual cortex. 
A
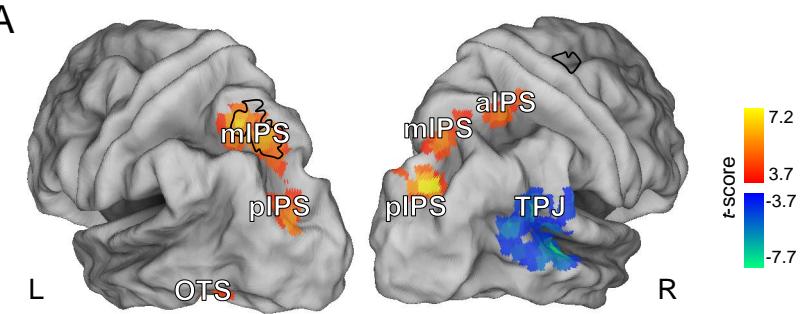

$\mathrm{B}$
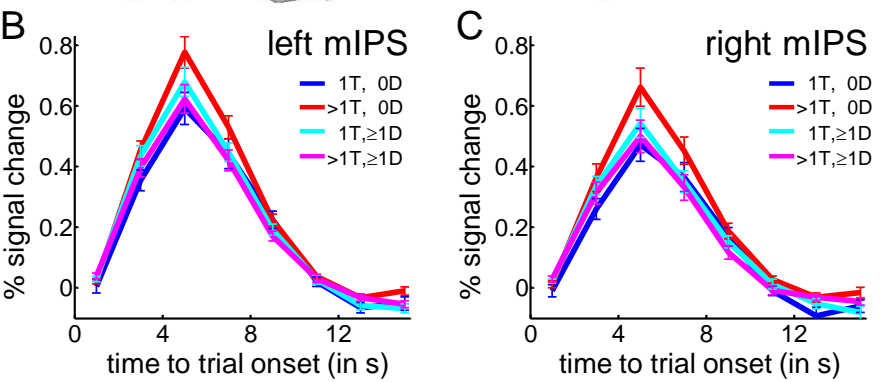

D

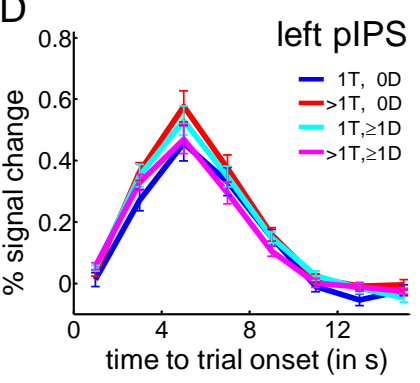

$\mathrm{E}$

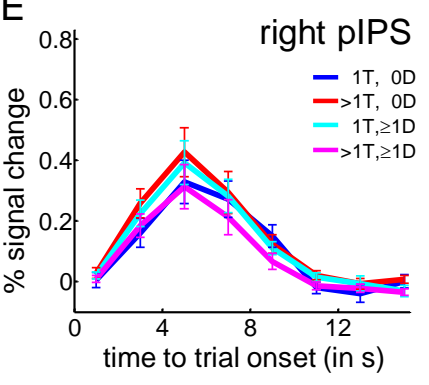

$\mathrm{F}$

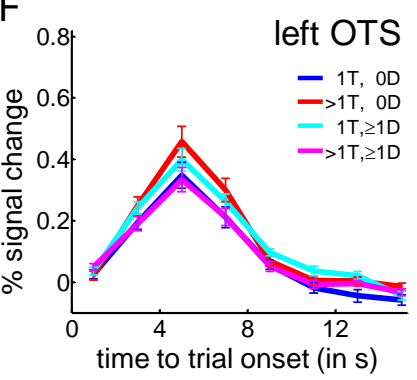

G
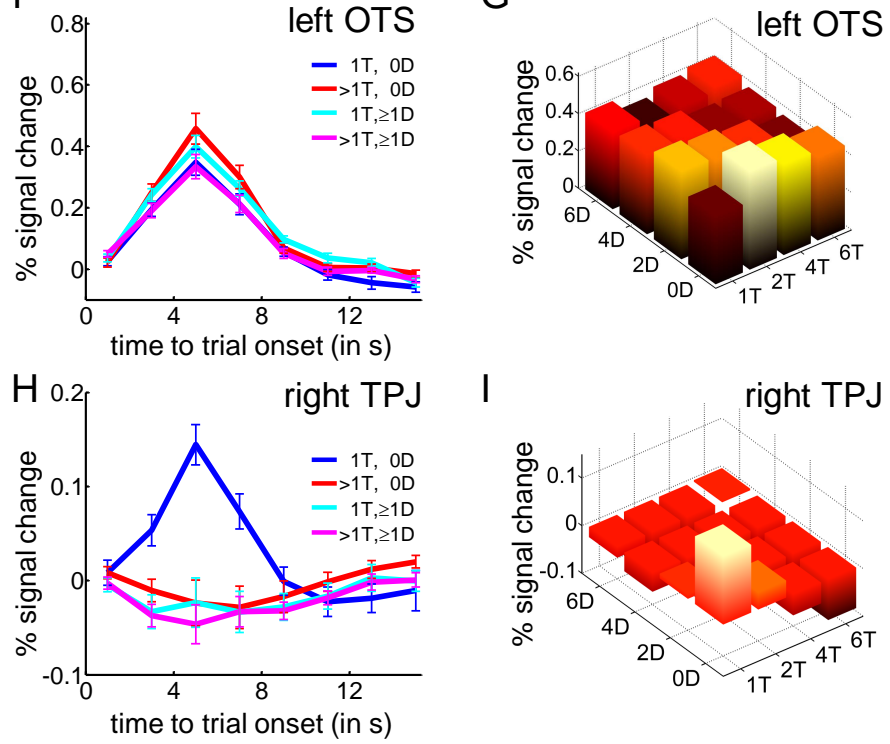

Figure 3 - Interaction between the difficulty of selection and access to VSTM (A) T-map (voxellevel uncorrected $P<0.001$, cluster-level FWE-corrected $P<0.05)$ for the interaction between target set size and distracter set size $[(>1 \mathrm{~T}, 0 \mathrm{D}$ minus $1 \mathrm{~T}, 0 \mathrm{D})$ minus $(>1 \mathrm{~T}, \geq 1 \mathrm{D}$ minus $1 \mathrm{~T} \geq 1 \mathrm{D})]$ (red to yellow) and the reverse (blue to cyan) (Huang and Pashler, 2007). The T-map is projected onto a surface rendering of the brain [PALS Atlas, Caret 5.612 (Van Essen, 2005)]. The black outline indicates which areas showed a significant main effect of adding targets to the display ( $>1 \mathrm{~T}$ minus $1 \mathrm{~T})$. (B-F,H) Time-activity curves averaged over all subjects and voxels belonging to the middle segment of left and right IPS (B-C), the posterior segment of left and right IPS (D-E), left OTS (F) and right TPJ (H). Error bars indicate 1 standard error of the mean across subjects. (G,I) Peak \% signal change for each condition in the $4 \times 4$ factorial design, averaged over all voxels belonging to left OTS (G) and right TPJ (I). The bars are color-scaled according to the response amplitude. Abbreviations: TPJ, temporoparietal junction; IPS, intraparietal sulcus; OTS, occipitotemporal sulcus. 


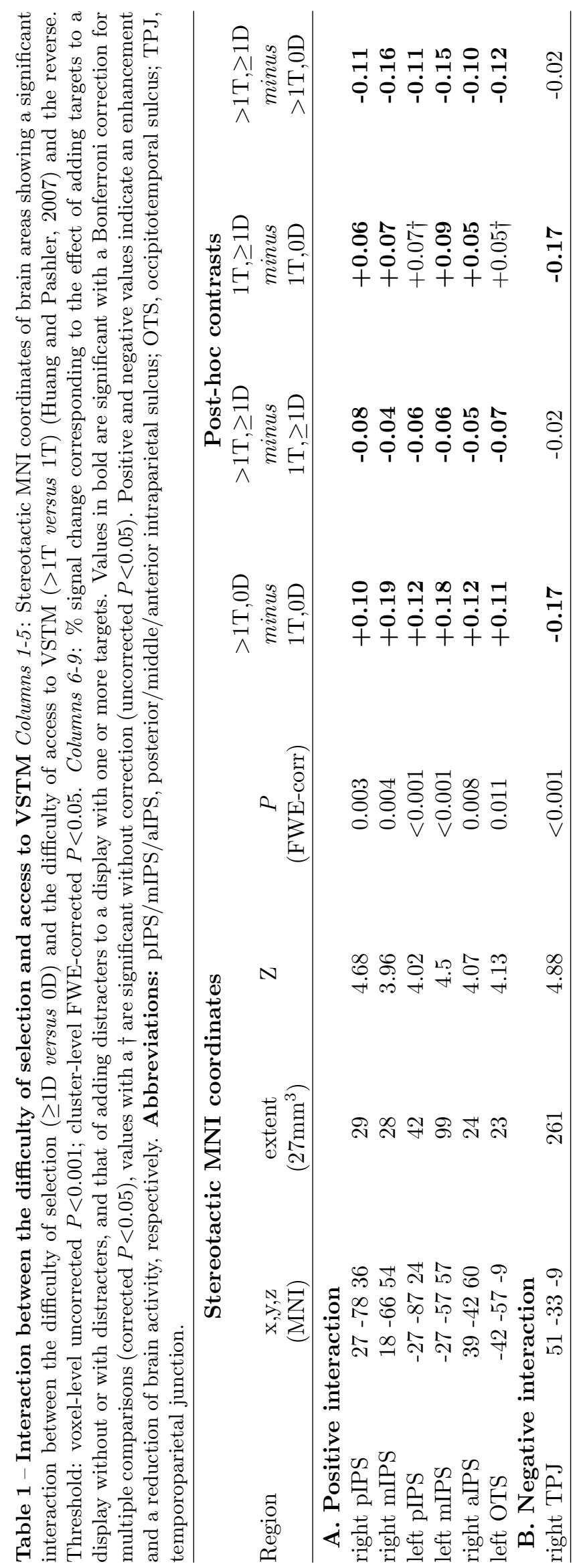




\subsubsection{TVA-based estimates of targets and distracters accessing VSTM}

Activity in left and right middle IPS (Fig. 4A-B), FEF and DMPFC linearly increased with the trial-by-trial estimates of the number of targets accessing VSTM (Table 2A). When lowering the threshold to an uncorrected $P<0.05$, we additionally observed a correlation between the number of targets in VSTM and activity in left $(-27,-84,30, Z=3.69$, uncorrected $P<0.01)$ and right posterior IPS $(30,-75,33, Z=2.00$, uncorrected $P<0.05)$. Conversely, activity in left and right angular gyrus (ANG) (Fig. 4A,C), among other regions, decreased with an increasing number of targets accessing VSTM (Table 2B).

Table 2 -fMRI: TVA-based single-trial predictions Stereotactic MNI coordinates of brain areas showing a significant linear modulation by the number of targets (A-B) and the number of distracters accessing VSTM (CD). Threshold: voxel-level uncorrected $P<0.001$; cluster-level FWE-corrected $P<0.05$. Abbreviations: FEF, frontal eye fields; IPS, intraparietal sulcus; DMPFC, dorsomedial prefrontal cortex; ANG, angular gyrus; LOC, lateral occipital complex; PCC, posterior cingulate cortex; SFG, superior frontal gyrus; MTG, middle temporal gyrus; IFG, inferior frontal gyrus.

\begin{tabular}{|c|c|c|c|c|}
\hline Region & $\begin{array}{c}\mathrm{x}, \mathrm{y}, \mathrm{z} \\
(\mathrm{MNI})\end{array}$ & $\begin{array}{c}\text { extent } \\
\left(27 \mathrm{~mm}^{3}\right)\end{array}$ & $\mathrm{Z}$ & $\begin{array}{r}P \\
(\mathrm{FWE}-\mathrm{corr}) \\
\end{array}$ \\
\hline \multicolumn{5}{|c|}{ A. Positive linear modulation by \# targets accessing VSTM } \\
\hline DMPFC & 92733 & 78 & 4.49 & $<0.001$ \\
\hline left IPS & $-21-6651$ & 18 & 3.77 & 0.051 \\
\hline right IPS & $15-6654$ & 49 & 4.17 & $<0.001$ \\
\hline left FEF & -24348 & 29 & 4.09 & 0.005 \\
\hline right $\mathrm{FEF}$ & 24657 & 70 & 5.23 & $<0.001$ \\
\hline \multicolumn{5}{|c|}{ B. Negative linear modulation by \# targets accessing VSTM } \\
\hline left ANG & $-45-6930$ & 149 & 4.41 & $<0.001$ \\
\hline right $\mathrm{ANG}$ & $45-6342$ & 34 & 3.72 & 0.002 \\
\hline left LOC & $-33-75-12$ & 53 & 4.06 & $<0.001$ \\
\hline right $\mathrm{LOC}$ & $36-84-9$ & 64 & 4.19 & $<0.001$ \\
\hline medial PCC & $-3-2733$ & 23 & 4.23 & 0.017 \\
\hline left SFG & -123651 & 76 & 4.1 & $<0.001$ \\
\hline right $\mathrm{MTG}$ & $63-486$ & 40 & 4.08 & 0.001 \\
\hline \multicolumn{5}{|c|}{ C. Positive linear modulation by \# distracters accessing VSTM } \\
\hline DMPFC & -32736 & 92 & 4.90 & $<0.001$ \\
\hline \multicolumn{5}{|c|}{ D. Negative linear modulation by \# distracters accessing VSTM } \\
\hline right $\mathrm{TPJ} / \mathrm{STG}$ & $66-4524$ & 801 & 5.28 & $<0.001$ \\
\hline right insula & $39-39$ & 24 & 4.55 & 0.009 \\
\hline right $\mathrm{MFG} / \mathrm{IFG}$ & 45915 & 145 & 4.52 & $<0.001$ \\
\hline left STS/STG & $-54-549$ & 114 & 4.4 & $<0.001$ \\
\hline right IFG & 48336 & 39 & 4.34 & $<0.001$ \\
\hline left SMG & $-63-2439$ & 20 & 3.73 & 0.023 \\
\hline
\end{tabular}

Second, when the average number of targets and distracters accessing VSTM per condition were used as independent variables, our general linear model yielded the following regression coefficients (Fig. 5A) (mean \pm s.e.m.): left IPS: $b_{0}=-0.67 \pm 0.15, b_{1}=0.21 \pm 0.05, b_{2}=0.13 \pm 0.05$; right IPS: $b_{0}=-1.14 \pm 0.24, b_{1}=0.29 \pm 0.10, b_{2}=0.18 \pm 0.10$. This model where targets and distracters 

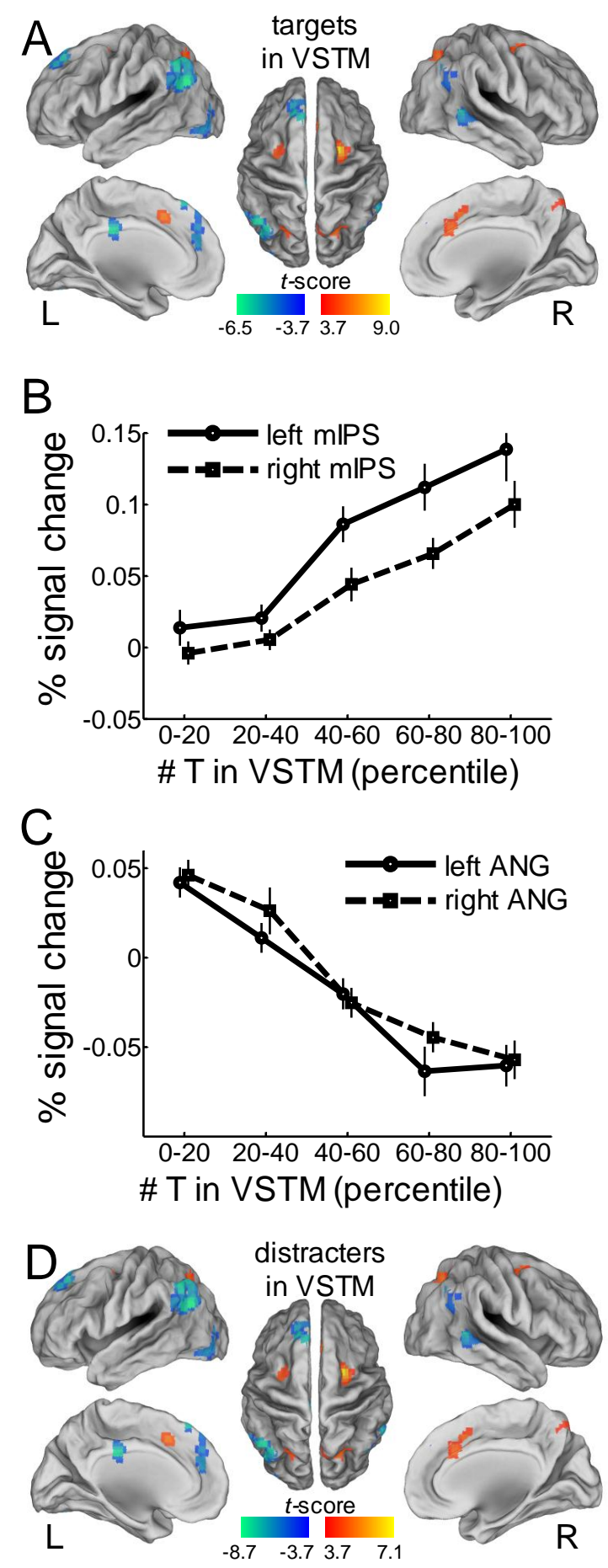

Figure 4 - fMRI: TVA-based single-trial predictions (A) T-map corresponding to a positive (red to yellow) and negative (blue to cyan) linear modulation by the number of targets accessing VSTM (voxel-level uncorrected $P<0.001$, cluster-level FWE-corrected $P<0.05)$. (B-C) Average effect size corresponding to the modulation by the number of targets accessing VSTM in left and right IPS (B), and left and right ANG (C). For each subject, the trials were ordered according to the value of the parametric regressor. The vector of the trials was then divided into five bins, each containing $20 \%$ of the trials. For example, the last bin (percentile 80 - percentile 100) contained the $20 \%$ trials with the highest value on the parametric regressor (Gläscher, 2009). Error bars indicate 1 standard error of the mean across subjects. (D) T-map corresponding to a positive (red to yellow) and negative (blue to cyan) linear modulation by the number of distracters accessing VSTM (voxel-level uncorrected $P<0.001$, cluster-level FWE-corrected $P<0.05)$. Abbreviations: IPS, intraparietal sulcus; ANG, angular gyrus. 
accessing VSTM receive differential weights resulted in a moderately good fit (Fig. 5B,D). In conditions however with a relatively high number of targets and distracters accessing VSTM the model resulted in a systematic overshoot (Fig. 5C,E).

\subsubsection{Analysis of interindividual differences in attentional parameters}

Interindividual differences in storage capacity $K$ were significantly correlated with activity in right middle IPS (Fig. 6A), DMPFC (Fig. 6B) and left and right FEF (Fig. 6C-D). No other correlations survived the statistical threshold.

\subsubsection{Control analyses}

Consistent with previous reports in the literature, a whole-brain analysis confirmed an effect of target set size in middle IPS when the display contained no distracters (Supplementary Fig. 2A-D) (Todd and Marois, 2004; Xu and Chun, 2006), and an effect of distracter set size when the display contained only a single target (Supplementary Fig. 2E-F) (Mitchell and Cusack, 2008; Molenberghs et al., 2008; Vandenberghe et al., 2005).

In order to establish whether or not the results were driven by the match or mismatch between the stimulus display and the probe display (Doricchi et al., 2010), we directly contrasted correct "change" and correct "no change" trials. The activity evoked by a matching probe was significantly larger than that evoked by a non-matching probe letter in right supramarginal gyrus, left and right basal ganglia and right insula (Supplementary Fig. 3). The cluster of activity in right supramarginal gyrus showed a small overlap with $3 \%$ (7 voxels) of the right TPJ cluster, but not with the right ANG cluster. The inverse contrast did not yield any significant result.

In a third control analysis, we evaluated the relationship between the number of stimuli on the display and the fMRI response amplitude. Activity in DMPFC increased linearly with an increasing number of stimuli on the display $(-3,30,36 ; Z=4.92$; extent $166 ; P<0.001)$. No effects of number of stimuli on display were seen in the posterior or middle IPS, even when the threshold was lowered to an uncorrected $P<0.05$. 


\section{A General Linear Model}

observed \% signal change $=b_{0}+b_{1}{ }^{*} E($ targets in VSTM $)+b_{2}{ }^{*} E($ distracters in VSTM $)+$ residual
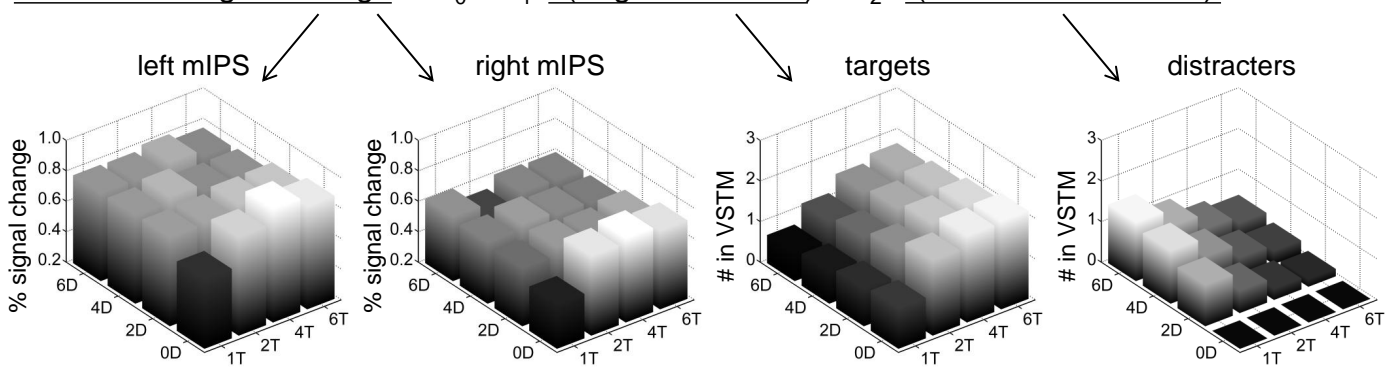

\section{$\%$ signal change in left mIPS}

B predicted based on model

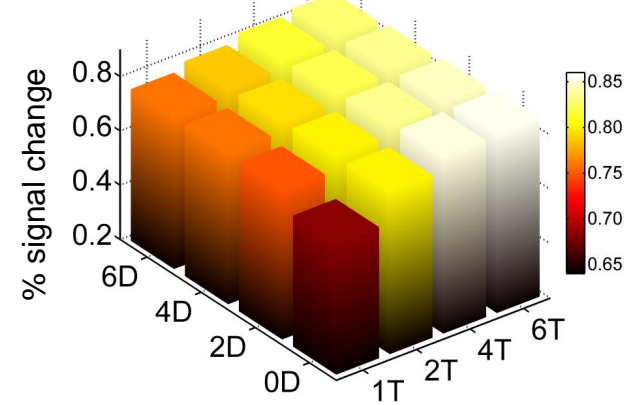

C residual: observed minus predicted

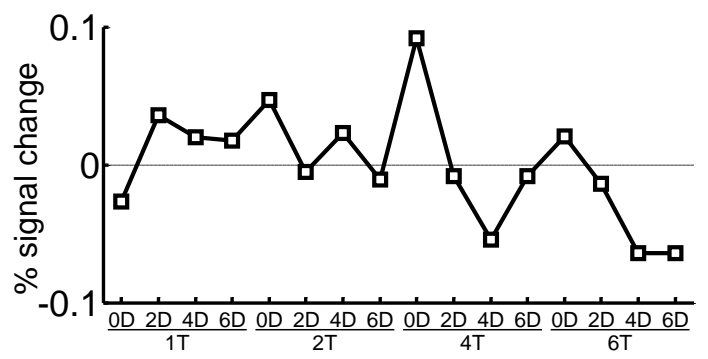

\section{\% signal change in right mIPS}

D predicted based on model

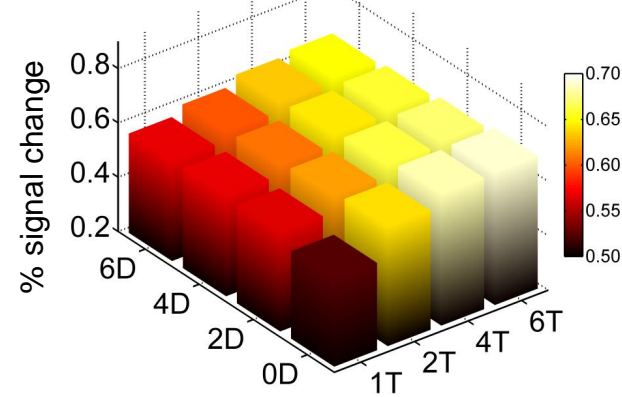

E residual: observed minus predicted

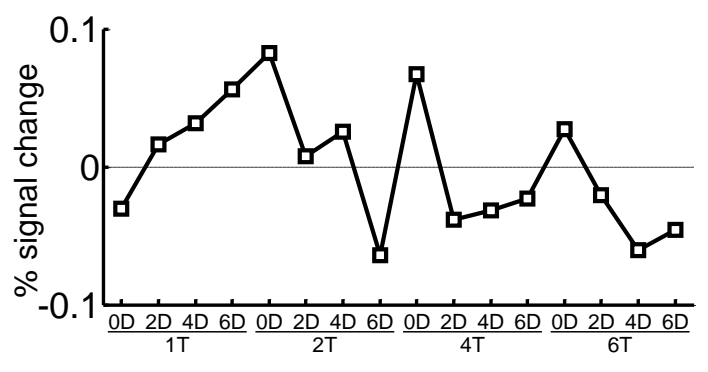

Figure 5 - fMRI: TVA-based predictions (A) General linear model with as dependent variables the observed peak \% signal change in left and right middle IPS for each subject and each of the 16 conditions, and with as independent variables the TVA-based estimates of targets and distracters accessing VSTM for each subject and each of the 16 conditions averaged over trials. (B) Peak \% signal change in the middle segment of left IPS, for each condition in the $4 \times 4$ factorial design, as predicted based on the general linear model: $b_{0}+b_{1} \mathrm{E}$ [Number of targets in VSTM] $+b_{2} \mathrm{E}$ [Number of distracters in VSTM]. (C). Residual: difference between the observed $\%$ signal change and the predicted $\%$ signal change in left middle IPS for each condition in the $4 \times 4$ factorial design. (D) Peak $\%$ signal change in the middle segment of right IPS, for each condition in the $4 \times 4$ factorial design, as predicted based on the general linear model: $b_{0}+b_{1} \mathrm{E}[$ Number of targets in VSTM $]+b_{2} \mathrm{E}[$ Number of distracters in VSTM]. (E). Residual: difference between the observed $\%$ signal change and the predicted $\%$ signal change in right middle IPS for each condition in the $4 \times 4$ factorial design. Abbreviations: mIPS, middle segment of the intraparietal sulcus 

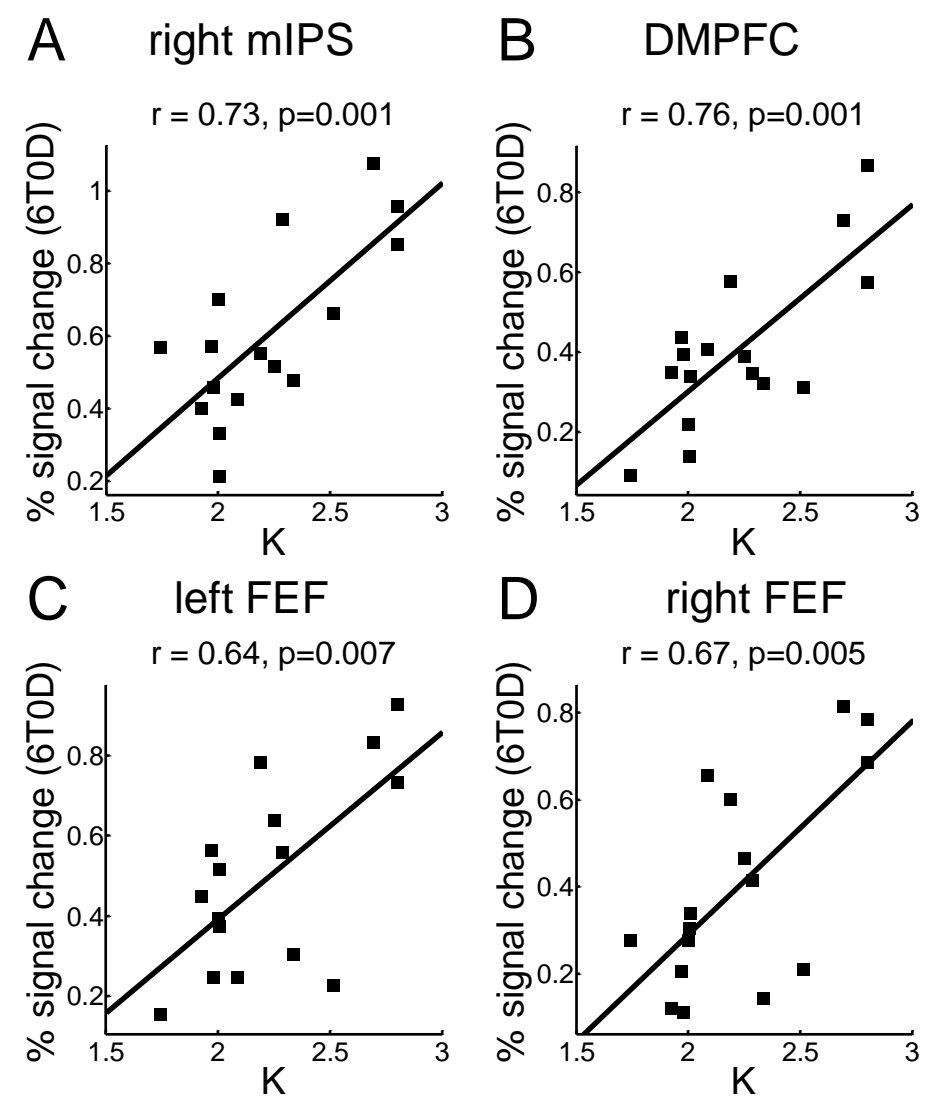

Figure 6 - Correlation between brain activity and attentional parameters (A-D) Correlation between $K$ and activity in right IPS (A), DMPFC (B), left FEF (C) and right FEF (D). The activity is expressed in \% signal change in the condition with a maximum proportion of targets (6T0D) relative to baseline. Abbreviations: IPS, intraparietal sulcus; DMPFC, dorsomedial prefrontal cortex; FEF, frontal eye fields 


\section{Discussion}

While we expected a significant main effect of both target set size and distracter set size, we mainly observed a strong interaction between target and distracter set size in both posterior and middle IPS (Fig. 2C-F; Fig. 3A-E): adding distracters to the display increased activity in case of a single target, whereas it suppressed activity in case of multiple targets. The activity increase in response to an increasing target set size disappeared in the presence of distracters. The inverse interaction was significant in right TPJ, which showed the highest response to target singletons (Fig. 3A,H-I).

Our data cannot be explained by sensory effects arising in early visual cortex. The stimulus array was only briefly presented and immediately followed by an effective mask covering all possible stimulus locations (Fig. 1A). As a consequence, we did not find effects of target or distracter set size on activity in early visual areas. Furthermore, our principal findings were based on interaction effects (Fig. 2C-F, 3) and sensory differences are subtracted from the interaction contrasts.

Neither can general effects of task difficulty account for any of the parietal response profiles: the pattern of accuracy differences between conditions (Fig. 1B-D) was qualitatively different from the pattern of response differences in parietal areas (Fig. 2D-G, 3I). The response profile of DMPFC, with a main effect of target and distracter set size but no interaction, however may reflect an increase in task difficulty (Barch et al., 1997).

Our estimates of $K$ are lower than those previously reported (Cowan, 2001; Luck and Vogel, 1997). This is likely due to the use of an unforced choice response procedure, which stimulates a conservative guessing strategy and improves the reliability of parameter estimates (Kyllingsbæk and Bundesen, 2009).

To maximize sensitivity of the fMRI response for effects of target and distracter set size, we did not manipulate exposure duration during the fMRI experiment. The estimation of the individual attentional parameters $(K, \alpha, \omega$ 's) during the fMRI experiment was therefore constrained by the perceptual threshold $\left(t_{0}\right)$ and processing speed $(C)$ derived from the behavioral experiment. This approach may have reduced the correlations between the attentional parameters and the fMRI response. The strength of the observed correlations (Fig. 6) suggests that the obtained parameters reliably capture individual differences in VSTM capacity and filtering efficiency.

Our experimental manipulation revealed a functional dissociation between 3 parietal ar- 
eas: the angular gyrus (Fig. 4A,C), the right temporoparietal junction (Fig. 3A,H-I), and the posterior and middle IPS segments bilaterally (Fig. 2, 3A-E). ANG belongs to a network of brain areas consistently deactivated in a wide range of cognitive tasks (Buckner et al., 2008), including working memory (Anticevic et al., 2010) and visual search tasks (Shulman et al., 2007). The response profile of ANG in our study was in agreement with these previous reports: ANG deactivation was more pronounced when the number of targets accessing VSTM was high (Fig. 4C).

Right TPJ and IPS responded to increasing target and distracter set size in a qualitatively different manner (Fig. 3I versus Fig. 2D-G). IPS showed activity increases when either targets or distracters were added to the display (Fig. 3B-E). In contrast, right TPJ was selectively activated when a single target was presented in the absence of any other targets or distracters (Fig. 3H-I). In that condition, the sudden onset of a single highly relevant perceptual unit may have captured attention (Yantis and Jonides, 1984, 1990). Under all other conditions, when attention was divided across multiple units in both hemifields, right TPJ was deactivated or did not significantly differ from baseline (Fig. 3I). It is important to note that a breach of expectancy (Arrington et al., 2000; Corbetta et al., 2000; Downar et al., 2000; Macaluso et al., 2002; Vossel et al., 2006) cannot explain the right TPJ activation: expectancy was matched between all conditions. Neither can it be accounted for by differential responses to matching versus non-matching probes (Doricchi et al., 2010): the activity levels in right TPJ were similar in correct "change" compared to correct "no change" trials. In a previous study, right TPJ activity correlated with the relative saliency of a target compared to other elements in the visual field (Mavritsaki et al., 2010). Activity levels of right TPJ are also increased during oddball trials (Braver et al., 2001; Bledowski et al., 2004; Downar et al., 2000). Taken together, these studies provide converging evidence that right TPJ may be involved in 'spotting target singletons', defined as targets which markedly and uniquely stand out against the background and are highly dissimilar from any other elements in the visual field. In natural vision, right TPJ may be recruited when a unique stimulus stands out against all other stimuli in the visual field by being perceptually or emotionally salient (Corbetta and Shulman, 2002; Corbetta et al., 2008).

With regards to the posterior and middle IPS, the overall response profiles were very similar between these two IPS segments (Fig. 2C-G). There however were some subtle differences: when the display contained multiple targets without distracters, activity levels in middle IPS were higher than when the display contained a single target with multiple distracters (Fig. 3B-C). In 
posterior IPS, these two conditions closely overlapped (Fig. 3D-E). The posterior IPS segment probably corresponds to what has been called IPS0/1 (Silver et al., 2005; Silver and Kastner, 2009; Swisher et al., 2007; Wandell et al., 2007), a visually responsive area that also shows strong effects of contralateral orienting in the presence of bilateral stimulation (Gillebert et al., 2011; Vandenberghe et al., 2005). Possibly, the posterior IPS segment is involved in attentional enhancement of stimuli within the contralateral hemispace at a more global spatial scale. In contrast, the middle IPS may code for attentional weights at the more specific level of individual units.

To our surprise, adding distracters to a multi-target array lowered IPS activity levels compared to an array consisting purely of targets. Likewise, increasing target set size abolished the enhancing effect of adding distracters. The resulting interaction between target and distracter set size (Fig. 2C-G, 3B-E) was an unpredicted and novel finding. A role of middle IPS in attentional selection between competing stimuli had led us to predict that the activity levels would be highest when selection demands were maximal, i.e. in the presence of multiple targets and distracters. This prediction was only confirmed when distracters were added to a single target. Neither can a simple account in terms of VSTM (Todd and Marois, 2004) account for the IPS findings (Fig. 1G compared to Fig. 2D-E): Although IPS reflects VSTM capacity relatively faithfully when the array exclusively consists of targets, as soon as multiple distracters are added the linear or asymptotic relationship between the number of units accessing VSTM (Fig. 1G) and the IPS activity pattern breaks down (Fig. 2D-E).

According to the Theory of Visual Attention (Bundesen, 1990; Bundesen et al., 2005), the number of items loaded into VSTM reach ceiling as targets and distracters increase above the maximal individual capacity (Fig. 1G). Assuming that the TVA-based predictions correctly reflect VSTM access, an additional operation beyond the mere number of items accessing VSTM (Todd and Marois, 2004) must be invoked to explain the IPS profile. Mathematically, we could approximate the middle IPS profile by giving differential weights to the contribution of targets versus distracters accessing VSTM (Fig 5B,D). Note that these differential weights for the VSTM phase differ from the attentional weights during the selection phase, which are already incorporated in the estimates of targets and distracters accessing VSTM (Bundesen and Habekost, 2008). But even when we allowed for such differential weighting, the model produced a substantial overshoot compared to the actual middle IPS responses when multiple targets and distracters were present (Fig 5C,E). Under conditions where multiple targets and distracters are simultaneously present, the IPS response cannot be explained purely based on VSTM: As 
target and distracter set size increased, activity was suppressed over and above what can be explained by differentially weighted targets and distracters accessing VSTM. Neither did the increased selection demands under these conditions lead to an IPS activity increase, in contrast to our a priori predictions. If the TVA-based VSTM estimates are correct, we have to conclude that during the selection phase multiple targets together with multiple distracters create a suppressive effect on IPS activity over and above any VSTM-related effects. In any case, the need to infer differential weights for targets and distracters within VSTM and the suppression elicited by high target and distracter set sizes call into question the neurobiological validity of the theoretical division between an attentional selection and a VSTM access phase as far as IPS is concerned.

Monkey electrophysiological studies may shed further light on the middle IPS profile: the lateral intraparietal area (LIP), the putative homologue of human middle IPS (Sereno et al., 2001; Vandenberghe and Gillebert, 2009), contains neurons whose firing rate increases when a relevant stimulus appears in their receptive field (Gottlieb et al., 1998), but is suppressed by an irrelevant stimulus (Ipata et al., 2006). Combining enhancement of target representation (Gottlieb et al., 1998) and distracter suppression (Falkner et al., 2010; Ipata et al., 2006) may result in a more sharply demarcated priority map and ultimately in the ability to better discriminate between targets and distracters. Although one has to be careful to infer fMRI response patterns from single neuron data, the middle IPS profile observed here (Fig. 3B-C) may reflect the interaction between enhancement and suppression at the level of the attentional priority map.

Other hypotheses could be put forward which we consider less likely as they would require one to modify the TVA-based predictions of targets and distracters entering VSTM themselves. For instance, for an array containing multiple targets and distracters, the threshold for VSTM access may be increased to prevent distracters from entering VSTM. A trade-off may exist between optimal access of targets to VSTM and restricted access of distracters. The optimal balance between liberal access for targets and restricted access for distracters may depend on factors such as the similarity between targets and distracters, the utility of targets for the observer, and the cost incurred by distracters entering VSTM. Such a cost could for instance arise if distracters in VSTM interfere with response decision processes (Allport, 1993). Alternatively, when multiple targets and distracters are present, the selection between targets and distracters prior to VSTM access may consume more resources so that fewer items reach the threshold for VSTM access under these taxing conditions. 


\section{Conclusions}

Overall, our results reflect the interaction between distinct attentional processes of biased competition (Bundesen, 1990; Bundesen et al., 2005; Desimone and Duncan, 1995). Selecting a target for access to VSTM is straightforward when the display contains one target and no other simultaneously competing and equally salient elements. TPJ may then act as a spotter of target singletons, increasing the amount of resources to the unique target stimulus. In contrast, when the display contains multiple relevant and irrelevant stimuli, particularly with high perceptual similarity, targets and distracters compete for access to VSTM. When multiple targets and distracters are displayed, the simple asymptotic relationship between number of items accessing VSTM and IPS activity levels breaks down. To approximate the IPS profile, targets and distracters accessing VSTM needed to be weighted differentially depending on their behavioral status (Fig. 5). Furthermore, an additional suppressive effect must be inferred to account for the relatively low activity levels under conditions of multiple targets and distracters. Our empirical findings call into question the neurobiological validity of the theoretical division between attentional selection and VSTM access as an account of IPS' response pattern. This reconciles the role of IPS in attentional selection between competing stimuli (Molenberghs et al., 2007, 2008; Vandenberghe et al., 2005, 2012) with its role in VSTM (Todd and Marois, 2004).

\section{Funding}

This work was supported by Research Foundation Flanders (FWO), Flanders, Belgium (grant number G0668.07 ESF EuroCores Program); Katholieke Universiteit Leuven (grant number OT/08/56) and Federaal Wetenschapsbeleid belspo (Inter-University Attraction Pole)(grant number $\mathrm{P} 6 / 29)$. CRG is a $\mathrm{PhD}$ fellow of the FWO and RV a senior clinical investigator of the FWO.

\section{Acknowledgements}

We are grateful to Professor Claus Bundesen and Jessie England for their comments and advice. 


\section{References}

Allport, A., 1993. Visual Attention. In: Posner, M. (Ed.), Foundations of Cognitive Science. Cambridge MA: MIT Press, pp. 631-682.

Anticevic, A., Repovs, G., Shulman, G.L., Barch, D.M., 2010. When less is more: TPJ and default network deactivation during encoding predicts working memory performance. Neuroimage 49, 2638-2648.

Arrington, C.M., Carr, T.H., Mayer, A.R., Rao, S.M., 2000. Neural mechanisms of visual attention: object-based selection of a region in space. J. Cogn. Neurosci. 12, 106-117.

Barch, D.M., Braver, T.S., Nystrom, L.E., Forman, S.D., Noll, D.C., Cohen, J.D., 1997. Dissociating working memory from task difficulty in human prefrontal cortex. Neuropsychologia $35,1373-1380$.

Beck, D.M., Kastner, S., 2009. Top-down and bottom-up mechanisms in biasing competition in the human brain. Vision. Res. 49, 1154-1165.

Bledowski, C., Prvulovic, D., Goebel, R., Zanella, F.E., Linden, D.E.J., 2004. Attentional systems in target and distractor processing: a combined ERP and fMRI study. Neuroimage $22,530-540$.

Braver, T.S., Barch, D.M., Gray, J.R., Molfese, D.L., Snyder, A., 2001. Anterior cingulate cortex and response conflict: effects of frequency, inhibition and errors. Cereb. Cortex 11, $825-836$.

Büchel, C., Holmes, A.P., Rees, G., Friston, K.J., 1998. Characterizing stimulus-response functions using nonlinear regressors in parametric fMRI experiments. Neuroimage 8, 140148.

Buckner, R.L., Andrews-Hanna, J.R., Schacter, D.L., 2008. The brain's default network: anatomy, function, and relevance to disease. Ann. N. Y. Acad. Sci. 1124, 1-38.

Bundesen, C., 1990. A theory of visual attention. Psychol. Rev. 97, 523-547.

Bundesen, C., Habekost, T., 2008. Principles of visual attention: Linking mind and brain. Oxford: Oxford University Press. 
Bundesen, C., Habekost, T., Kyllingsbæk, S., 2005. A neural theory of visual attention: bridging cognition and neurophysiology. Psychol. Rev. 112, 291-328.

Corbetta, M., Kincade, J.M., Ollinger, J.M., McAvoy, M.P., Shulman, G.L., 2000. Voluntary orienting is dissociated from target detection in human posterior parietal cortex. Nat. Neurosci. 3, 292-297.

Corbetta, M., Patel, G., Shulman, G.L., 2008. The reorienting system of the human brain: from environment to theory of mind. Neuron 58, 306-324.

Corbetta, M., Shulman, G., 2002. Control of goal-directed and stimulus-driven attention in the brain. Nat. Rev. Neurosci. 3, 201-215.

Cowan, N., 2001. The magical number 4 in short-term memory: a reconsideration of mental storage capacity. Behav. Brain Sci. 24, 87-114.

Desimone, R., Duncan, J., 1995. Neural mechanisms of selective visual attention. Annu. Rev. Neurosci. 18, 193-222.

Doricchi, F., Macci, E., Silvetti, M., Macaluso, E., 2010. Neural correlates of the spatial and expectancy components of endogenous and stimulus-driven orienting of attention in the Posner task. Cereb. Cortex 20, 1574-1585.

Downar, J., Crawley, A.P., Mikulis, D.J., Davis, K.D., 2000. A multimodal cortical network for the detection of changes in the sensory environment. Nat. Neurosci. 3, 277-283.

Duncan, J., 1996. Cooperating brain systems in selective perception and action. In: Inui, I., McClelland, J.L. (Eds.), Attention and performance XVI, Cambridge: The MIT Press. pp. $549-578$.

Dyrholm, M., Kyllingsbæk, S., Espeseth, T., Bundesen, C., 2011a. Generalizing parametric models by introducing trial-by-trial parameter variability: The case of TVA. Journal of Mathematical Psychology 55, 416-429.

Dyrholm, M., Kyllingsbæk, S., Vangkilde, S., Habekost, T., Bundesen, C., 2011b. Single trial inference on visual attention. In: Pham, T.D., Zhou, X., Tanaka, H., Oyama-Higa, M., Jiang, X., Sun, C., Kowalski, J., Jia, X. (Eds.), International Symposium on Computational Models for Life Sciences, pp. 37-43. 
Falkner, A.L., Krishna, B.S., Goldberg, M.E., 2010. Surround suppression sharpens the priority map in the lateral intraparietal area. J. Neurosci. 30, 12787-12797.

Gazzaley, A., 2011. Influence of early attentional modulation on working memory. Neuropsychologia 49, 1410-1424.

Gillebert, C.R., Mantini, D., Thijs, V., Sunaert, S., Dupont, P., Vandenberghe, R., 2011. Lesion evidence for the critical role of the intraparietal sulcus in spatial attention. Brain 134, 16941709.

Gläscher, J., 2009. Visualization of group inference data in functional neuroimaging. Neuroinformatics $7,73-82$.

Gottlieb, J.P., Kusunoki, M., Goldberg, M.E., 1998. The representation of visual salience in monkey parietal cortex. Nature 391, 481-484.

Habekost, T., Bundesen, C., 2003. Patient assessment based on a theory of visual attention (TVA): subtle deficits after a right frontal-subcortical lesion. Neuropsychologia 41, 1171-1188.

Huang, L., Pashler, H., 2007. A Boolean map theory of visual attention. Psychol. Rev. 114, 599-631.

Hung, J., Driver, J., Walsh, V., 2005. Visual selection and posterior parietal cortex: Effects of repetitive transcranial magnetic stimulation on partial report analyzed by Bundesen's Theory of Visual Attention. J. Neurosci. 25, 9602-9612.

Ipata, A.E., Gee, A.L., Gottlieb, J., Bisley, J.W., Goldberg, M.E., 2006. LIP responses to a popout stimulus are reduced if it is overtly ignored. Nat. Neurosci. 9, 1071-1076.

Kastner, S., Ungerleider, L.G., 2001. The neural basis of biased competition in human visual cortex. Neuropsychologia 39, 1263-1276.

Kyllingsbæk, S., 2006. Modeling visual attention. Behav. Res. Methods. 38, 123-133.

Kyllingsbæk, S., Bundesen, C., 2009. Changing change detection: improving the reliability of measures of visual short-term memory capacity. Psychon. Bull. Rev. 16, 1000-1010.

Lavie, N., 2005. Distracted and confused? Selective attention under load. Trends Cogn. Sci. 9, $75-82$. 
Lepsien, J., Griffin, I.C., Devlin, J.T., Nobre, A.C., 2005. Directing spatial attention in mental representations: Interactions between attentional orienting and working-memory load. Neuroimage 26, 733-743.

Lepsien, J., Nobre, A.C., 2007. Attentional modulation of object representations in working memory. Cereb. Cortex 17, 2072-2083.

Lepsien, J., Thornton, I., Nobre, A.C., 2011. Modulation of working-memory maintenance by directed attention. Neuropsychologia 49, 1569-1577.

Luck, S.J., Vogel, E.K., 1997. The capacity of visual working memory for features and conjunctions. Nature 390, 279-281.

Macaluso, E., Frith, C.D., Driver, J., 2002. Supramodal effects of covert spatial orienting triggered by visual or tactile events. J. Cogn. Neurosci. 14, 389-401.

Macmillan, N., Creelman, C., 1991. Detection theory: A user's guide. Cambridge, UK: Cambridge University Press.

Mavritsaki, E., Allen, H.A., Humphreys, G.W., 2010. Decomposing the neural mechanisms of visual search through model-based analysis of fMRI: top-down excitation, active ignoring and the use of saliency by the right TPJ. Neuroimage 52, 934-946.

McNab, F., Klingberg, T., 2008. Prefrontal cortex and basal ganglia control access to working memory. Nat. Neurosci. 11, 103-107.

Mitchell, D.J., Cusack, R., 2008. Flexible, capacity-limited activity of posterior parietal cortex in perceptual as well as visual short-term memory tasks. Cereb. Cortex 18, 1788-1798.

Molenberghs, P., Gillebert, C.R., Peeters, R., Vandenberghe, R., 2008. Convergence between lesion-symptom mapping and functional magnetic resonance imaging of spatially selective attention in the intact brain. J. Neurosci. 28, 3359-3373.

Molenberghs, P., Mesulam, M.M., Peeters, R., Vandenberghe, R., 2007. Re-mapping attentional priorities: Differential contribution of superior parietal lobule and intraparietal sulcus. Cereb. Cortex 17, 2703-2712.

Peers, P.V., Ludwig, C.J.H., Rorden, C., Cusack, R., Bonfiglioli, C., Bundesen, C., Driver, J., Antoun, N., Duncan, J., 2005. Attentional functions of parietal and frontal cortex. Cereb. Cortex 15, 1469-1484. 
Poline, J.B., Worsley, K.J., Evans, A.C., Friston, K.J., 1997. Combining spatial extent and peak intensity to test for activations in functional imaging. Neuroimage 5, 83-96.

Sereno, M.I., Pitzalis, S., Martinez, A., 2001. Mapping of contralateral space in retinotopic coordinates by a parietal cortical area in humans. Science 294, 1350-1354.

Shibuya, H., Bundesen, C., 1988. Visual selection from multielement displays: measuring and modeling effects of exposure duration. J. Exp. Psychol. Hum. Percept. Perform. 14, 591-600.

Shulman, G.L., Astafiev, S.V., McAvoy, M.P., d'Avossa, G., Corbetta, M., 2007. Right TPJ deactivation during visual search: functional significance and support for a filter hypothesis. Cereb. Cortex 17, 2625-2633.

Silver, M.A., Kastner, S., 2009. Topographic maps in human frontal and parietal cortex. Trends Cogn. Sci. 13, 488-495.

Silver, M.A., Ress, D., Heeger, D.J., 2005. Topographic maps of visual spatial attention in human parietal cortex. J. Neurophysiol. 94, 1358-1371.

Swisher, J.D., Halko, M.A., Merabet, L.B., McMains, S.A., Somers, D.C., 2007. Visual topography of human intraparietal sulcus. J. Neurosci. 27, 5326-5337.

Todd, J.J., Marois, R., 2004. Capacity limit of visual short-term memory in human posterior parietal cortex. Nature 428, 751-754.

Todd, J.J., Marois, R., 2005. Posterior parietal cortex activity predicts individual differences in visual short-term memory capacity. Cogn. Affect. Behav. Neurosci. 5, 144-155.

Van Essen, D.C., 2005. A population-average, landmark- and surface-based (PALS) atlas of human cerebral cortex. Neuroimage 28, 635-662.

Vandenberghe, R., Geeraerts, S., Molenberghs, P., Lafosse, C., Vandenbulcke, M., Peeters, K., Peeters, R., Hecke, P.V., Orban, G., 2005. Attentional responses to unattended stimuli in human parietal cortex. Brain 128, 2843-2857.

Vandenberghe, R., Gillebert, C.R., 2009. Parcellation of human parietal cortex: converging evidence from functional imaging in the intact human brain and patient lesion studies. Behav. Brain Res. 199, 171-182. 
Vandenberghe, R., Molenberghs, P., Gillebert, C.R., 2012. Spatial attention deficits in humans: The critical role of superior compared to inferior parietal lesions. Neuropsychologia .

Vossel, S., Thiel, C.M., Fink, G.R., 2006. Cue validity modulates the neural correlates of covert endogenous orienting of attention in parietal and frontal cortex. Neuroimage 32, 1257-1264.

Wager, T.D., Nichols, T.E., 2003. Optimization of experimental design in fMRI: a general framework using a genetic algorithm. Neuroimage 18, 293-309.

Wandell, B.A., Dumoulin, S.O., Brewer, A.A., 2007. Visual field maps in human cortex. Neuron $56,366-383$.

Xu, Y., Chun, M.M., 2006. Dissociable neural mechanisms supporting visual short-term memory for objects. Nature 440, 91-95.

Yantis, S., Jonides, J., 1984. Abrupt visual onsets and selective attention: evidence from visual search. J. Exp. Psychol. Hum. Percept. Perform. 10, 601-621.

Yantis, S., Jonides, J., 1990. Abrupt visual onsets and selective attention: voluntary versus automatic allocation. J. Exp. Psychol. Hum. Percept. Perform. 16, 121-134. 